\title{
Laser Acupuncture Reduces Body Fat in Obese Female Undergraduate Students
}

\author{
Xiao-Guang Liu, Juan Zhang, Jian-Liang Lu, and Timon Cheng-Yi Liu
}

Laboratory of Laser Sports Medicine, South China Normal University,

Guangzhou 510006, China

Correspondence should be addressed to Xiao-Guang Liu, tygx@jnu.edu.cn

Received 8 February 2012; Revised 19 April 2012; Accepted 21 April 2012

Academic Editor: Rui Duan

Copyright ( $) 2012$ Xiao-Guang Liu et al. This is an open access article distributed under the Creative Commons Attribution License, which permits unrestricted use, distribution, and reproduction in any medium, provided the original work is properly cited.

The purpose of this study was to investigate the efficacies of laser acupunctures with different laser irradiation doses for body weight loss and body fat reduction in obese persons. Twentyeight subjects with simple obesity were divided into four groups receiving laser acupunctures at $0,358,478$, and $597 \mathrm{~J} / \mathrm{cm}^{2}$, respectively, which were applied to the acupoints of Cv 8 , St 25 , and Sp 15, six times per week for four weeks. Body weight (BW), body mass index (BMI), body fat mass (BFM), body fat percent, waist girth, hip girth, and waist-to-hip ratio (WHR) were measured before and after treatment. The laser groups receiving 358 and $597 \mathrm{~J} / \mathrm{cm}^{2}$ showed significant reductions in BW, BMI, BFM, waist girth, hip girth, and WHR after treatment compared with the placebo group. The percent reductions of BFM in the laser groups receiving 358 and $597 \mathrm{~J} / \mathrm{cm}^{2}$ were $4.29 \%$ and $3.94 \%$, respectively, and the corresponding values of BW were $1.99 \%$ and $1.63 \%$, respectively. In conclusion, laser acupuncture could reduce body fat mass and lower body weight in a dose-dependent manner in obese persons. Among the doses of 358, 478, and $597 \mathrm{~J} / \mathrm{cm}^{2}$, both the 358 and $597 \mathrm{~J} / \mathrm{cm}^{2}$ doses were effective in the 4-week trial.

\section{Introduction}

With economic growth and constant urbanization, the prevalence of obesity has been increasing in many countries. Obesity is associated with many chronic diseases, including cardiovascular diseases, diabetes, musculoskeletal diseases, and some cancers. Common intervention methods for obesity include low-calorie diet, exercise, behavioral modification, and slimming drugs [1]. Also acupuncture has shown good therapeutic results in the treatment of obesity [2].

Over the last 30 years, low-level laser therapy has been applied in many medical fields, and low-level laser irradiation has been adopted in acupuncture [3]. Laser acupuncture was found to be effective in the treatment of visceral postmenopausal obesity when combined with lowcalorie diet [4]. A recent clinical observation showed that laser acupuncture alone could reduce body weight (BW) and body mass index (BMI) in obese persons [5], but the study lacked a placebo control. Moreover, the influence of laser acupuncture on body composition was unclear, and further scientific evidence for the efficacy of laser acupuncture for obesity should be presented.

In this study, we conducted a blinded clinical trial to investigate the efficacies of laser acupunctures with GaAlAs laser irradiation at different doses for body weight loss and body fat reduction in obese humans.

\section{Materials and Methods}

The study protocol was approved by the ethics committee of the Faculty of South China Normal University, Guangzhou, China. Informed consent was obtained from each participant prior to the start of treatment.

2.1. Subjects and Groups. Twentyeight female undergraduate students with simple obesity aged 19 25 years were enrolled in this study at the Laboratory of Laser Sports Medicine of South China Normal University in Guangzhou. Based on the obesity criteria in the Asian-Pacific region recommended by the World Health Organization [6], inclusion criteria for 
TABLE 1: Body weight and body mass index data in the placebo group and the three laser groups before and after treatment.

\begin{tabular}{lcccc}
\hline Measurement & Group & Pretreatment & Posttreatment & Change over time \\
\hline \multirow{3}{*}{ BW $(\mathrm{kg})$} & Placebo & $64.43 \pm 5.79$ & $64.51 \pm 5.89$ & $-0.09 \pm 0.72$ \\
& Low-dose laser & $71.76 \pm 13.34$ & $70.33 \pm 12.37^{*}$ & $1.43 \pm 1.37^{\Delta}$ \\
& Medium-dose laser & $71.64 \pm 11.24$ & $70.83 \pm 11.45^{*}$ & $0.81 \pm 0.76$ \\
& High-dose laser & $77.49 \pm 16.91$ & $76.23 \pm 16.36^{*}$ & $1.26 \pm 1.33^{\Delta}$ \\
BMI $\left(\mathrm{kg} / \mathrm{m}^{2}\right)$ & Placebo & $26.94 \pm 1.62$ & $27.00 \pm 1.83$ & $-0.06 \pm 0.28$ \\
& Low-dose laser & $28.31 \pm 3.53$ & $27.68 \pm 3.38^{*}$ & $0.63 \pm 0.51^{\Delta \Delta}$ \\
& Medium-dose laser & $27.93 \pm 3.54$ & $27.61 \pm 3.67^{*}$ & $0.31 \pm 0.31$ \\
\hline
\end{tabular}

BW: body weight, BMI: body mass index. Asterisks indicate significant differences from pretreatment $\left({ }^{*} P<0.05\right)$. Triangles indicate significant differences from the placebo group $\left({ }^{\Delta} P<0.05,{ }^{\Delta} P<0.01\right)$.

the subjects were determined as body mass index (BMI) $\geq 25 \mathrm{~kg} / \mathrm{m}^{2}$ and body fat percent $\geq 30 \%$. All subjects were randomly divided into four groups, namely, placebo group, low-dose laser group, medium-dose laser group, and highdose laser group, seven in each group. Each subject was asked not to change her diet or exercise habits during the trial.

2.2. Laser Acupuncture. A GaAlAs laser (Model LD-1, Guangzhou, China) with $810 \mathrm{~nm}$ wavelength, $0 \sim 500 \mathrm{~mW}$ adjustable and continuous power output, and with $0.4 \mathrm{~cm}$ beam diameter was used. The subjects in the placebo group, the low-dose group, the medium-dose group, and the highdose group were treated with the laser at the doses of 0 , 358,478 , and $597 \mathrm{~J} / \mathrm{cm}^{2}$ (powers: $0,150,200$, and $250 \mathrm{~mW}$; intensity: 0, 1194, 1592, and $1990 \mathrm{~mW} / \mathrm{cm}^{2}$; irradiation time: $5 \mathrm{~min} /$ point, $25 \mathrm{~min}$ in total), respectively, six times per week for four weeks. The following abdominal acupuncture points were irradiated: Cv 8 (Shenque), St 25 (Tianshu, bilateral), and Sp 15 (Daheng, bilateral). The laser irradiations were applied directly to the skin of the points with a perpendicular beam.

2.3. Outcome Measures. BW, BMI, body fat mass (BFM), body fat percent, waist girth, hip girth, and waist-to-hip ratio (WHR) were measured at baseline and the end of treatment. BW, BMI, BFM, and body fat percent were measured using the body composition analyzer (Model Inbody 3.0, Republic of Korea). Waist girth was measured at the level of the umbilicus, and hip girth was measured at the level of maximum extension of the buttocks. WHR was calculated as waist girth divided by hip girth.

2.4. Statistical Analysis. Data were expressed as mean \pm standard deviation. Differences between before and after treatment in each group were analyzed by paired $t$-tests. Differences between group means were analyzed using oneway ANOVA with post hoc test. The statistical level of significance was set at $P<0.05$.

\section{Results}

3.1. Body Weight and Body Composition. There were no significant differences in BW, BMI, BFM, and body fat percent at baseline between any groups (Tables 1 and 2). After the four weeks of treatment, the three laser groups exhibited significant drops in BW, BMI, and BFM except the medium-dose group in BFM, while the placebo group exhibited no significant changes. Mean BWs in the lowdose group, the medium-dose group, and the high-dose group were reduced by $1.43,0.81$, and $1.26 \mathrm{~kg}$ (percent reductions: $1.99 \%, 1.13 \%$, and $1.63 \%$ ), respectively. Mean BMIs in the three laser groups decreased by $0.63,0.31$, and $0.53 \mathrm{~kg} / \mathrm{m}^{2}$, respectively (Table 1 ). Mean BFMs in the three laser groups were reduced by $1.16,0.69$, and $1.16 \mathrm{~kg}$ (percent reductions: $4.29 \%, 2.60 \%$, and $3.94 \%$ ), respectively (Table 2). The changes in BW, BMI, and BFM in the lowdose and high-dose groups were greater than those in the placebo group with statistical significance, but there were no significant differences between the medium-dose group and the placebo group. There were no significant differences in body weight and body composition after treatment between any laser groups. In addition, each laser group showed a nonsignificant drop in body fat percent after treatment.

3.2. Waist Girth and Hip Girth. There were no significant differences in waist girth, hip girth, and WHR at baseline between any groups (Table 3). After the four weeks of treatment, the low-dose and high-dose groups showed significant drops in waist girth and WHR. Each laser group also showed a nonsignificant drop in hip girth after treatment. Mean waist girths in the low-dose group and the high-dose group were reduced by 2.34 and $2.14 \mathrm{~cm}$, respectively. Mean WHR in each laser group decreased by 0.01 . The changes in waist girth, hip girth, and WHR in the low-dose and highdose groups were greater than those in the placebo group with statistical significance, but there were no significant differences between the medium-dose group and the placebo group. There were no significant differences in both the girths after treatment between any laser groups.

\section{Discussion}

Obesity is a result of disturbance of energy homeostasis. Acupuncture and low-level laser irradiation with their homeostatic activities may give a chance to primarily compensate for the condition. According to traditional Chinese medicine, 
TABLE 2: Body fat mass and body fat percent data in the placebo group and the three laser groups before and after treatment.

\begin{tabular}{lcccc}
\hline Measurement & Group & Pretreatment & Posttreatment & Change over time \\
\hline \multirow{3}{*}{ BFM $(\mathrm{kg})$} & Placebo & $24.00 \pm 3.84$ & $23.97 \pm 3.58$ & $0.03 \pm 0.66$ \\
& Low-dose laser & $27.06 \pm 7.60$ & $25.90 \pm 6.87^{*}$ & $1.16 \pm 1.11^{\Delta}$ \\
& Medium-dose laser & $26.57 \pm 7.36$ & $25.88 \pm 7.50$ & $0.69 \pm 0.97$ \\
\hline High-dose laser & $29.47 \pm 10.53$ & $28.31 \pm 9.92^{*}$ & $1.16 \pm 1.02^{\Delta}$ \\
\hline BF\% & Placebo & $37.07 \pm 2.89$ & $37.07 \pm 2.62$ & $0.00 \pm 0.80$ \\
& Low-dose laser & $37.34 \pm 4.33$ & $36.48 \pm 4.19$ & $0.86 \pm 0.94$ \\
& Medium-dose laser & $36.64 \pm 5.47$ & $36.04 \pm 5.54$ & $0.60 \pm 1.04$ \\
\hline
\end{tabular}

BFM: body fat mass, BF\%: body fat percent. Asterisks indicate significant differences from pretreatment $\left({ }^{*} P<0.05\right)$. Triangles indicate significant differences from the placebo group $\left({ }^{\triangle} P<0.05\right)$.

TABLE 3: Waist girth, hip girth, and waist-to-hip ratio data in the placebo group and the three laser groups before and after treatment.

\begin{tabular}{|c|c|c|c|c|}
\hline Measurement & Group & Pretreatment & Posttreatment & Change over time \\
\hline \multirow{4}{*}{ WG $(\mathrm{cm})$} & Placebo & $87.71 \pm 6.10$ & $88.21 \pm 6.53$ & $-0.50 \pm 1.22$ \\
\hline & Low-dose laser & $90.77 \pm 12.23$ & $88.43 \pm 11.07^{*}$ & $2.34 \pm 1.96^{\Delta \Delta}$ \\
\hline & Medium-dose laser & $90.03 \pm 7.57$ & $88.64 \pm 7.88$ & $1.39 \pm 1.92$ \\
\hline & High-dose laser & $91.59 \pm 12.13$ & $89.44 \pm 11.61^{*}$ & $2.14 \pm 1.75^{\Delta \Delta}$ \\
\hline \multirow{4}{*}{$\mathrm{HG}(\mathrm{cm})$} & Placebo & $101.71 \pm 5.64$ & $101.93 \pm 5.41$ & $-0.21 \pm 0.70$ \\
\hline & Low-dose laser & $104.79 \pm 6.47$ & $103.93 \pm 5.83$ & $0.86 \pm 0.94^{\triangle}$ \\
\hline & Medium-dose laser & $105.64 \pm 9.16$ & $105.14 \pm 9.13$ & $0.50 \pm 0.50$ \\
\hline & High-dose laser & $105.93 \pm 9.68$ & $105.29 \pm 9.55$ & $0.64 \pm 0.85^{\triangle}$ \\
\hline \multirow{4}{*}{ WHR } & Placebo & $0.86 \pm 0.05$ & $0.87 \pm 0.06$ & $0.00 \pm 0.01$ \\
\hline & Low-dose laser & $0.86 \pm 0.07$ & $0.85 \pm 0.07^{*}$ & $0.01 \pm 0.01^{\Delta}$ \\
\hline & Medium-dose laser & $0.85 \pm 0.04$ & $0.84 \pm 0.04$ & $0.01 \pm 0.02$ \\
\hline & High-dose laser & $0.86 \pm 0.04$ & $0.85 \pm 0.04^{*}$ & $0.01 \pm 0.01^{\Delta}$ \\
\hline
\end{tabular}

WG: waist girth, HG: hip girth, WHR: waist-to-hip ratio. Asterisks indicate significant differences from pretreatment $(* P<0.05)$. Triangles indicate significant differences from the placebo group $\left({ }^{\circ} P<0.05, \Delta \Delta P<0.01\right)$.

all afflictions of obesity primarily originate from energy deficiency in the meridians St (stomach) and Sp (spleen). With classic needle stimulating some acupoints in the meridians St and Sp, many acupuncture therapies for obesity have shown good results [7-9].

Laser acupuncture integrates the positive effects of acupuncture and low-level laser irradiation. Compared with classic needle acupuncture, laser acupuncture manifests many advantages: aseptic and painless stimulation of acupoints, possibility of application to some needle-forbidden acupoints such as Cv 8 (Shenque) with no subsequent transient erythema, and lack of tissue traumatization. Only a few papers dealing with laser acupuncture referred to its application in the treatment of obesity. Wozniak et al. [4] administered laser acupuncture at the acupoints of $\mathrm{Cv}$ 12 (Zhongwan), St 25 (Tianshu), and St 36 (Zusanli) in postmenopausal women with visceral obesity and found that laser acupuncture could increase the efficacy of low-calorie diet for obesity. Hu et al. [5] treated 95 patients of simple obesity with laser acupuncture at the acupoints of $\mathrm{Cv} 9$ (Shuifen), St 25 (Tianshu), St 28 (Shuidao), St 40 (Fenglong), and Sp 15 (Daheng) with $785 \mathrm{~nm}$ pulse laser irradiation. In the trial, they did not use placebo control, and their results showed that laser acupuncture alone could reduce both BW and BMI without restrictive diet. Adopting a placebo control, we presented convincing evidence for the efficacy of laser acupuncture alone for obesity in this study. According to the pathogeny of simple obesity in traditional Chinese medicine and clinical data of acupuncture therapy for obesity, we chose $\mathrm{Cv} 8$, St 25, and Sp 15 to treat obesity in the laser acupuncture research. Our results showed that laser acupuncture with the doses of 358 and $597 \mathrm{~J} / \mathrm{cm}^{2}$ significantly reduced both BW and BMI in obese subjects compared with the placebo control. In this study, the effect of laser acupuncture on body composition in female obese undergraduate students was investigated. Our results showed that the laser acupuncture with $810 \mathrm{~nm}$ GsAlAs laser irradiation at both the low dose of $358 \mathrm{~J} / \mathrm{cm}^{2}$ and the high dose of $597 \mathrm{~J} / \mathrm{cm}^{2}$ significantly reduces BFM as well as BW in obese subjects. In the lowdose and high-dose groups, the percent reduction was greater in BFM than in BW (in the low-dose group, $4.29 \%$ versus $1.99 \%$; in the high-dose group, $3.94 \%$ versus $1.63 \%$ ). This result indicated that laser acupuncture lowered BW primarily by reducing BFM, but not water. The mechanism by which laser acupuncture reduced BFM may include the systemic response and local response. Acupoint of $\mathrm{Cv} 8$ is located at the centre of the umbilicus, which is underlain by the inferior epigastric artery and vein. When laser acupuncture 
was applied at $\mathrm{Cv} 8$, low-level laser irradiation may exert a systemic regulating effect on fat metabolism through influencing the blood in the inferior epigastric artery and vein. In this study, hip girth losses in the low-dose and high-dose groups were probably a consequence of systemic regulation of body fat by laser acupuncture. Neira et al. [10] in 2002 reported that low-level laser therapy could release stored fat in adipocytes by the opening of cell membraneassociated pores after a few minutes treatment. Caruso-Davis et al. [11] confirmed the finding of Neira et al. [10] in the investigation of the efficacy of low-level laser therapy for spot fat reduction and at the same time found that low-level laser irradiation over the waist could significantly reduce waist girth in healthy adults, which is a typical phenomenon of indirect photobiomodulation [12]. Acupoints of St 25 and Sp 15 are located in abdominal area with thicker fat at the level the umbilicus. Therefore, waist girth losses after laser acupuncture at St 25 and Sp 15 may partly result from the direct effect of low-level laser irradiation on fat tissue in this study.

We also observed the dose-response relationship of laser acupuncture therapy for obesity in this study. Based on data of several trials of GaAlAs laser therapy $[13,14]$, we adopted the doses of 358,478 , and $597 \mathrm{~J} / \mathrm{cm}^{2}$ to conduct the laser acupuncture research. The irradiations at both $358 \mathrm{~J} / \mathrm{cm}^{2}$ and $597 \mathrm{~J} / \mathrm{cm}^{2}$ significantly reduced all the measurements except body fat percent compared with placebo irradiation, whereas the irradiation at $478 \mathrm{~J} / \mathrm{cm}^{2}$ nonsignificantly did. Among the three doses, the lowest dose of $358 \mathrm{~J} / \mathrm{cm}^{2}$ appeared to be the best efficient in the laser acupuncture therapy, but there were no significant differences in all the measurements between any laser groups. There are various factors which may influence the efficacy of laser acupuncture therapy for obesity, including laser parameters, skin properties, and subjects' behavior of exercise. Thus, more studies were required to definitely show the dose-response pattern of laser acupuncture therapy for obesity. In addition, the irradiations at all the three doses nonsignificantly reduced body fat percent. The nonsignificant changes in the three laser groups might become significant with the extension of treatment period according to the self-adaptive property of low-level laser irradiation [15]. Of course, it should be further verified.

\section{Conclusion}

Laser acupuncture could reduce body fat mass and lower body weight in a dose-dependent manner in obese persons. Among the doses of 358,478 , and $597 \mathrm{~J} / \mathrm{cm}^{2}$, both the 358 and $597 \mathrm{~J} / \mathrm{cm}^{2}$ doses were effective in the 4 -week trial.

\section{Conflict of Interests}

The authors declare that there is no conflict of interests.
(2011B031600006) and National Natural Science Foundation of China (60878061).

\section{References}

[1] R. H. Eckel, "Clinical practice. Nonsurgical management of obesity in adults," The New England Journal of Medicine, vol. 358, no. 18, pp. 1886-1950, 2008.

[2] S. H. Cho, J. S. Lee, L. Thabane, and J. Lee, "Acupuncture for obesity: a systematic review and meta-analysis," International Journal of Obesity, vol. 33, no. 2, pp. 183-196, 2009.

[3] P. Whittaker, "Laser acupuncture: past, present, and future," Lasers in Medical Science, vol. 19, no. 2, pp. 69-80, 2004.

[4] P. Wozniak, G. Stachowiak, A. Piêta-Doliñska, and P. Oszukowski, "Laser acupuncture and low-calorie diet during visceral obesity therapy after menopause," Acta Obstetricia et Gynecologica Scandinavica, vol. 82, no. 1, pp. 69-73, 2003.

[5] W. L. Hu, C. H. Chang, and Y. C. Hung, "Clinical observations on laser acupuncture in simple obesity therapy," American Journal of Chinese Medicine, vol. 38, no. 5, pp. 861-867, 2010.

[6] World Health Organization, Western Pacific Region, and International Association for the Study of Obesity, The AsiaPacific Perspective: Redefining Obesity and Its Treatment, Health Communications Australia Pty Limited, Sydney, Australia, 2000.

[7] Q. Shan, "Acupuncture therapy for 130 cases of simple obesity," Journal of Acupuncture and Tuina Science, vol. 4, no. 2, pp. 121-122, 2006.

[8] M. J. Mu and Y. Q. Yuan, "Clinical study on simple obesity treated with abdomen acupuncture," Journal of Acupuncture and Tuina Science, vol. 6, no. 3, pp. 165-168, 2008.

[9] Y. Shi, C. Zhao, and X. Y. Zuo, "Clinical study on treatment of simple obesity due to spleen deficiency by acupuncturemoxibustion," Journal of Acupuncture and Tuina Science, vol. 6, no. 6, pp. 352-355, 2008.

[10] R. Neira, J. Arroyave, H. Ramirez et al., "Fat liquefaction: effect of low-level laser energy on adipose tissue," Plastic and Reconstructive Surgery, vol. 110, no. 3, pp. 912-922, 2002.

[11] M. K. Caruso-Davis, T. S. Guillot, V. K. Podichetty et al., "Efficacy of low-level laser therapy for body contouring and spot fat reduction," Obesity Surgery, vol. 21, no. 6, pp. 722729, 2011.

[12] T. C. Y. Liu, E. X. Wei, and F. H. Li, "Indirect photobiomodulation on tumors," Lasers in Surgery and Medicine, vol. 44, supplement 24, 2012.

[13] R. T. Chow, L. B. Barnsley, and G. Z. Heller, "The effect of $300 \mathrm{~mW}, 830 \mathrm{~nm}$ laser on chronic neck pain: a double-blind, randomized, placebo-controlled study," Pain, vol. 124, no. 12, pp. 201-210, 2006.

[14] U. Dundar, D. Evcik, F. Samli, H. Pusak, and V. Kavuncu, "The effect of gallium arsenide aluminum laser therapy in the management of cervical myofascial pain syndrome: a double blind, placebo-controlled study," Clinical Rheumatology, vol. 26, no. 6, pp. 930-934, 2007.

[15] T. C. Y. Liu, L. Luo, and L. Zhang, "Self-adaptive effects of low intensity laser irradiation in prophylaxis of muscular fibrosis," Lasers in Surgery and Medicine, vol. 43, supplement 23, article 975, 2011.

\section{Acknowledgments}

This work was supported by the Science and Technology Planning Foundation of Guangdong Province, China 


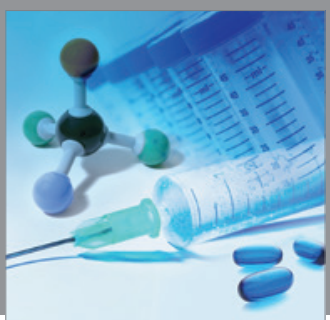

International Journal of

Medicinal Chemistry

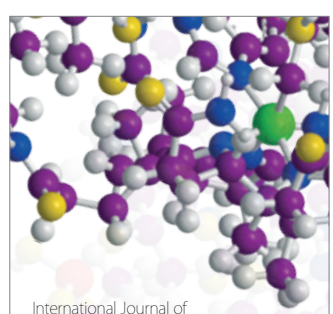

Carbohydrate Chemistry

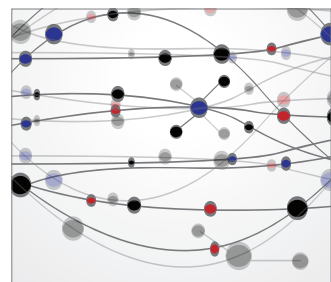

The Scientific World Journal
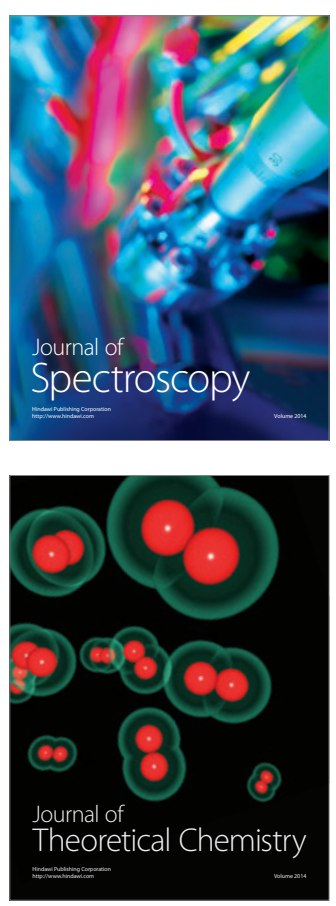
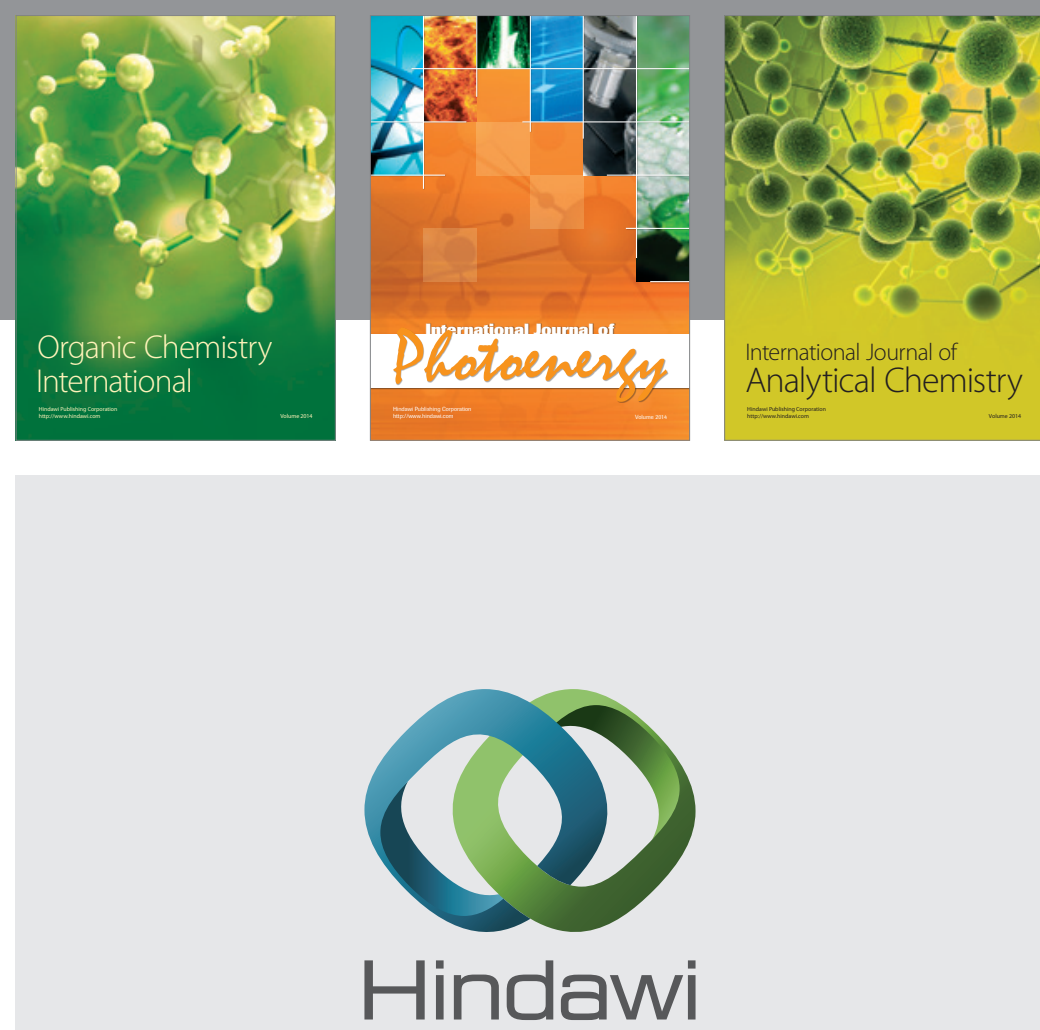

Submit your manuscripts at

http://www.hindawi.com
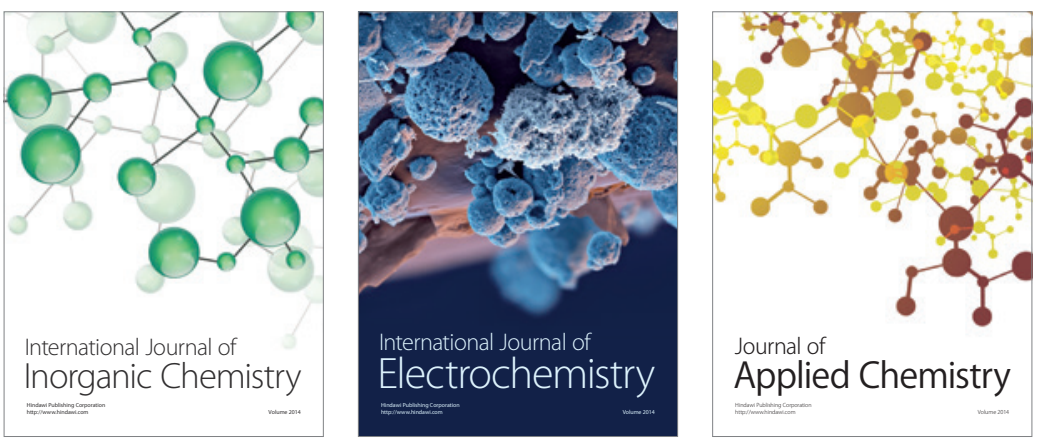

Journal of

Applied Chemistry
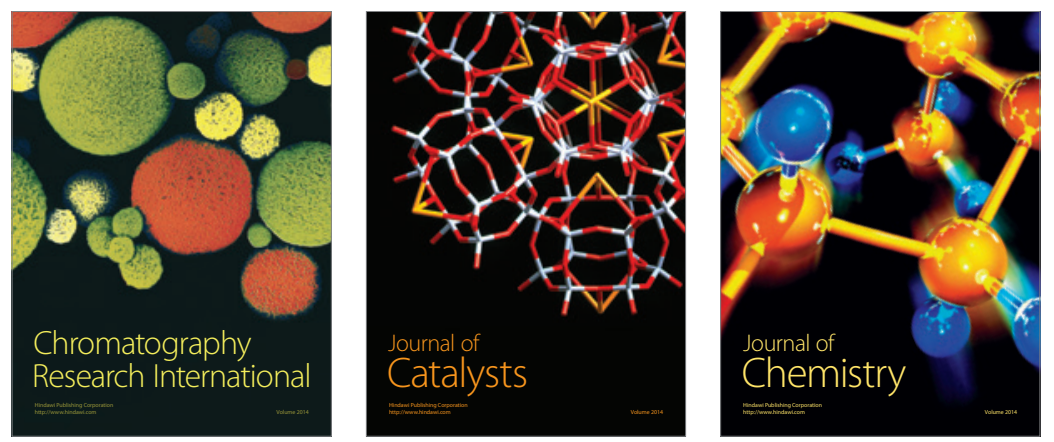
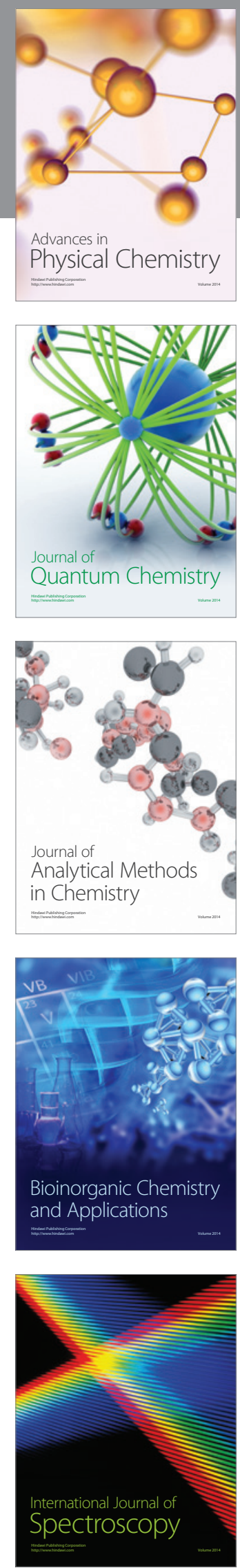